\title{
V1493 AQL AN EXTREMELY DISTANT NOVA?
}

\author{
A. Dobrotka, ${ }^{1}$ A. Retter, ${ }^{2}$ L. Hric,${ }^{3}$ R. Novak, ${ }^{4}$ O. Shemmer, ${ }^{5}$ and Y. Lipkin ${ }^{5}$
}

\begin{abstract}
Analysis of CCD photometry performed during 12 nights and the estimate of the distance based on the light curve is presented. The photometric data is modulated with a period of $3.7 \mathrm{~h}$, which we interpret as the orbital period above the period gap distribution of cataclysmic variables. The VSNET light curve combined with data from IAU Circulars suggests a distance of $4.5 \pm 0.4 \mathrm{kpc}$ based on $t_{2} / t_{3}$ time estimates. Two spectra 11 days after the maximum were taken. The expansion velocity of the envelope was derived to be $1658 \pm 62$ $\mathrm{km} \mathrm{s}^{-1}$.
\end{abstract}

Nova Aql 1999 (V1493 Aql) was discovered by Akihiko Tago, Tsuyama on July $13^{\text {th }} 1999$ (Nakano et al. 1999). The nova reached a maximum of $\sim$ $8.8 \mathrm{mag}$ in V. By Tomov et al. (1999) the expansion velocity is $\sim 1700 \mathrm{~km} \mathrm{~s}^{-1}$. Bonifacio et al. (2000) derived $A_{V}$ to be $1.04 \pm 0.3 \mathrm{mag}$ and they estimated the distance of the nova to be $18.8 \pm 3.6$ kpc. They concluded that in such an extreme case the nova should be on the boundary or outside our galaxy. By Arkhipova et al. (2002) $A_{\mathrm{V}}=5.03 \pm 0.3$ mag and the distance is $4.2 \mathrm{kpc}$.

To estimate the distance we used the empirical fornulas (MMRD) for times $t_{2}$ and $t_{3}$. Both time parameters were derived from the light curves based on the data published in IAUC and from the VSNET (Fig. 1). The mean value of derived distances is $4.5 \pm$ $0.4 \mathrm{kpc}$. We suppose the extinction by Arkhipova et al. to be more realistic. Hence we do not agree with the interpretation of Bonifaccio et al. (2000).

\footnotetext{
${ }^{\mathrm{I}}$ Departement of Physics, Faculty of Materials Science and Technology, Slovak University of Technology in Bratislava, Paulinska 16, Trnava 917 24, The Slovak Republic(dobrotka@ mtf.stuba.sk).

${ }^{2}$ School of Physics, University of Sydney, 2006, Australia, (e-mail: retter@physics.usyd.edu.au).

${ }^{3}$ Astronomical Institute of the Slovak Academy of Sciences, 05960 Tatranská Lomnica, The Slovak Republic, (email: hric(@ita.3.sk).

${ }^{4}$ Nicholas Copernicus Observatory and Planetarium in Brno, Kravi hora 2,616 00 Brno, Czech Republic, (e-mail: novak(whvezdarna.cz).

${ }^{5}$ The Raymond and Beverly Sackler faculty of exact sciences, school of physics and astronomy and the Wise Observatory, Tel-Aviv University, Israel, (e-mail: ohad@wise.tau.ac.il, yiftah(u)wise.tau.ac.il).
}

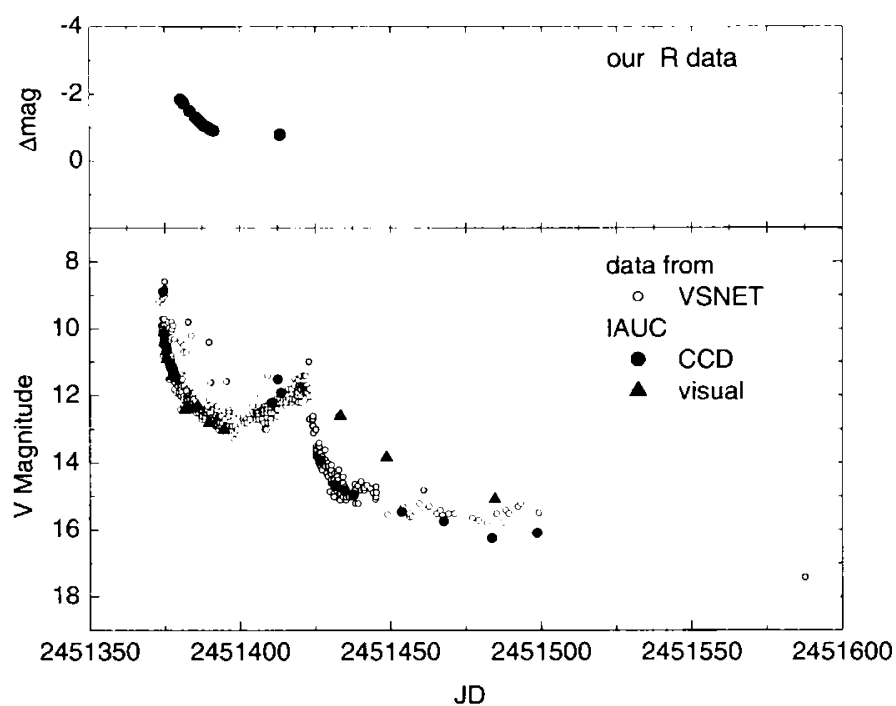

Fig. 1. The light curve of V1493 Aql in the R and V band. Upper panel: our data; Bottom panel: VSNET and IAUC data.

Our own photometry is made by CCD camera ST-7 in R filter at the Nicholas Copernicus Observatory and a single run was conducted with the $1.0-\mathrm{m}$ telescope in I filter at Wise Observatory (WO) Israel, on 1999 Aug $1^{\text {st }}$. Spectroscopy of V1494 Aql was carried out at WO in July $24^{\text {th }} 1999$. These data are unsuitable to complete the used light curves. Our runs were used for period analysis. We have found a period of $3.7 \mathrm{~h}$, which we interpret as the orbital period.

Besides the Balmer series He II 4686 is evident. The strongest $\mathrm{Fe}$ lines are Fe II 5019, 5169 and the forbidden line [Fe VII] 5721. The next probable forbidden line is [O I] 5580. Another Oxygen line is O I 7777. The spectrum shows the Na I 5890 doublet and probably a blend of O VI 5292 with [Ca V] 5309. No line shows $\mathrm{P}$ Cygni profile. The expansion velocity of the envelope was derived to be $1658 \pm 62$ $\mathrm{km} \mathrm{s}^{-1}$

\section{REFERENCES}

Arkhipova, V.P., Burlak, M.A., Esipov, V.F., 2002, AL 28,100

Bonifacio, P., Selvelli, P.L., Caffau, E., 2000, A\&A 356, L53

Nakano, S., Tago, A., 1999, IAUC 7223

Tomov, T., Moro. D., Munari, U., 1999, IAUC 7225 